\title{
Exciton Exchange between Nearly-Free and Acceptor-Bound States of a Positive Trion Assisted by Cyclotron Excitation
}

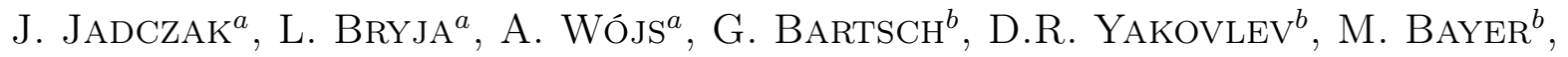 \\ P. PlochockA ${ }^{c}$, M. Potemski $^{c}$, D. Reuter ${ }^{d}$ And A. Wieck ${ }^{d}$ \\ ${ }^{a}$ Institute of Physics, Wrocław University of Technology, Wyb. Wyspiańskiego 27, 50-370 Wrocław, Poland \\ ${ }^{b}$ Experimentelle Physik 2, Technische Universität Dortmund, D-44227 Dortmund, Germany \\ ${ }^{c}$ Grenoble High Magnetic Field Laboratory, CNRS, 38-042 Grenoble, France \\ ${ }^{d}$ Lehrstuhl fur Festkörperphysik, Ruhr Universität, Universitätstr., Bochum, Germany
}

\begin{abstract}
In magneto-photoluminescence spectra of a two-dimensional hole gas in a GaAs quantum well we observe coupling of two different radiative states. The pair of coupled states are an acceptor-bound trion $\mathrm{AX}^{+}$and an essentially free (only weakly localized by a shallow lateral potential) trion $\mathrm{X}^{+}$, brought into resonance by an additional cyclotron excitation controlled by the magnetic field. The coupling mechanism is the exciton transfer, and the optical signature is a clear anticrossing of the emission lines of an $\mathrm{X}^{+}$and a cyclotron replica of the $\mathrm{AX}^{+}$.
\end{abstract}

PACS: 73.20.Mf, 71.35.Ji, 71.35.Pq, 78.20.Ls

\section{Introduction}

In the recent years two-dimensional electron and hole systems in high magnetic fields have been intensively studied in the context of many-body effects associated with the Coulomb interaction among the carriers also in optical experiments [1]. Most previous experiments were focused on the electrons, as the hole systems usually had a lower carrier mobility and a more complicated structure of the valence band. However, with gradual improvement of the quality of hole systems, their analysis also became increasingly valueable, especially for those effects aided by a larger effective mass [2,3]. The subject of the present paper is an intriguing anticrossing observed in the magneto-photoluminescence (PL) spectrum, understood as the coupling of essentially-free and acceptor-bound trion states immersed in a magnetically quantized hole gas, aided by an additional cyclotron excitation.

\section{Experimental results}

The studied sample was a $22 \mathrm{~nm}$ wide GaAs/ $\mathrm{Ga}_{0.65} \mathrm{Al}_{0.35} \mathrm{As}$ quantum well, grown by molecular beam epitaxy on a (001) semi-insulating GaAs substrate, $\delta$ carbon doped in the barrier on one side. The well was separated from the doping layer by a $40 \mathrm{~nm}$ wide spacer. The hole mobility measured at $T=4.2 \mathrm{~K}$ was equal to $\mu=1.71 \times 10^{5} \mathrm{~cm}^{-2} /(\mathrm{Vs})$. The hole concentration measured at low temperatures $(T=17 \mathrm{mK}-4.2 \mathrm{~K})$ and estimated from the quantum Hall effect measured in van der Pauw configuration, was almost temperature independent. In the dark, it was equal to $p=1.43 \times 10^{11} \mathrm{~cm}^{-2}$. Under laser illumination it decreased linearly with excitation power density, by up to several percent. The PL was excited by the $632.8 \mathrm{~nm}$ line of a helium-neon laser (above the band gap of the barrier). The optical experiments were performed at $T=2.3 \mathrm{~K}$, in the Faraday configuration, in magnetic fields up to $B=17 \mathrm{~T}$ varied with a small step $\Delta B=0.05 \mathrm{~T}$. The switch between the $\sigma^{-}$and $\sigma^{+}$helices was achieved by means of reversing the direction of the field. The spectra were analyzed in a high-resolution monochromator, with a nitrogen-cooled 2048-pixel CCD camera.

The magnetic field evolution of PL spectrum in $\sigma$ polarization is presented in Fig. 1. In the absence of a field a single line is observed. When magnetic field is applied, this line splits into two distinct components. The energy positions of both lines can be resolved in the fields above $B=6 \mathrm{~T}$. We attribute the observed lines to the neutral exciton $\mathrm{X}$ (higher energy) and the spin-singlet state of a positive trion $\mathrm{X}_{\mathrm{s}}^{+}$(lower energy).

In the lower energy part of the spectrum we detect the whole group of lines of very weak intensity. Two of them depart from the remaining ones toward higher energy, linearly with the increase of the magnetic field (with the slope of $\approx 1.0 \mathrm{meV} / \mathrm{T}$ ). This effect is similar to the "shake-up" process [4] observed previously also by us [3] in a symmetric $15 \mathrm{~nm}$ wide GaAs quantum well, but here the cyclotron shift goes in the opposite energy direction. The magnetic field slope for the rest of weak lines (not marked in Fig. 1) is about $0.65 \mathrm{meV} / \mathrm{T}$. All of these weak lines are interpreted as follows: those lines with the smaller energy-vs.-field slope are due to the recombination of the $\mathrm{AX}^{+}$complexes (consisting of a trion bound to an acceptor positioned inside the well), whereas the two other lines with the higher slope are their hole cyclotron replicas, here called CR-AX ${ }_{1}^{+}$and CR-AX ${ }_{2}^{+}$(in analogy to a previous observation of an electron cyclotron replica of the free exciton line [5], there emerging on the high-energy side of the free negative trions). The pres- 
ence of a pair of $\mathrm{CR}-\mathrm{AX}^{+}$replicas is a consequence of the fact that the allowed (by the conservation of angular momentum and spin) recombination of the $\mathrm{AX}^{+}$ground state (labeled by the $3 \mathrm{~h}$ spin of $1 / 2$ and the total $3 \mathrm{~h}+\mathrm{e}$ angular momentum projection of -1 ) occurs to a pair of spin-singlet and triplet states of the $\mathrm{A}^{+}$, separated by an exchange gap. Comparison of the slopes of CR-AX ${ }^{+}$and $\mathrm{AX}^{+}$yields a difference of $0.35 \mathrm{meV} / \mathrm{T}$, which agrees very well with the hole cyclotron energy determined earlier from the cyclotron resonance of the low-density GaAs/ AlGaAs quantum wells [6] (interpolation of those results for the $22 \mathrm{~nm}$ width gives $0.28 \mathrm{meV} / \mathrm{T})$. The effective mass corresponding to the measured slope is $m_{\mathrm{h}}=0.33$.

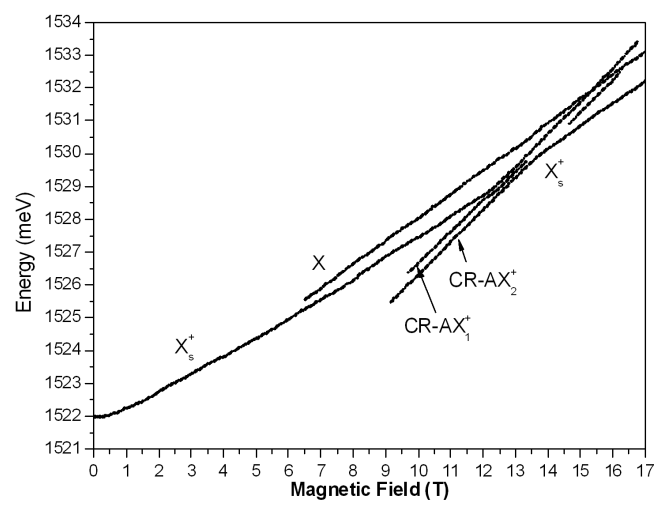

Fig. 1. Energy position of all observed lines in photoluminescence in $s^{-}$polarization.

The most intriguing effect is observed when the pair of $\mathrm{CR}-\mathrm{AX}^{+}$lines approach the positive trion $\mathrm{X}_{\mathrm{s}}^{+}$which results in a clear anticrossing. Furthermore, the intensity of both $\mathrm{CR}-\mathrm{AX}^{+}$lines is greatly enhanced in the narrow anticrossing region. Beyond this region all lines return to a similar behavior as in the lower fields (both in terms of energy and intensity).

Interpretation of this anticrossing was aided by the numerical calculation of the energy and recombination spectra of the involved radiative complexes [3, 7]. The conclusion is that the $\mathrm{X}^{+}$and $\mathrm{AX}^{+}$states are brought into resonance by an additional cyclotron excitation of a nearby free hole. The coupling mechanism for this pair of quasi-degenerate states is the resonant exciton transfer between the localized and (essentially) free trion states. The coupling strength (dependent on, e.g., the relative acceptor and hole concentrations) extracted from the energy splitting at the point of anticrossing is about $0.15 \mathrm{meV}$. The coincidence of the degeneracy of the initial radiative states $\mathrm{X}^{+}$and $\mathrm{AX}^{+}$(required for their efficient mixing) and of the transition energies (whose anticrossing has been directly observed in the PL spectrum) is explained by the near degeneracy of the optically active $\mathrm{A}^{+}$channel (corresponding to the angular momentum -1 of the $\mathrm{AX}^{+}$ground state) with the free hole state, the two serving as the final states in the radiative recombination of either $\mathrm{X}^{+}$or $\mathrm{AX}^{+}[3]$. The above interpretation is well supported by a simple calculation involving the set of constant $\mathrm{X}^{+}$and $\mathrm{AX}^{+}$oscillator strengths and the linear field dependences of the uncoupled initial and final states (extracted from the spectrum outside of the anticrossing region) and the constant coupling strength (determined at the point of anticrossing).

\section{Conclusion}

We have detected the anticrossing of the (essentially) free trion lines with a pair of cyclotron replicas of an acceptor-bound trion line. The effect has been observed here in a 2D hole gas; it has not been earlier detected in an electron system (partly due to a less favorable electron effective mass). Its explanation involves a resonant exciton exchange between free and bound trions assisted by the cyclotron hole excitation.

\section{Acknowledgments}

This work has been supported by the research grant No. N202179538 of the Polish MNiSW, Marie Curie Grant No. PIEF-GA-2008-221701 of EU (AW) and EC-EuroMagNetII No. 228043-CNRS-PICS-4340.

\section{References}

[1] D. Heiman, B.B. Goldberg, A. Pinczuk, C. W. Tu, A.C. Gossard, J. H. English, Phys. Rev. Lett. 61, 605 (1988); B.B. Goldberg, D. Heiman, A. Pinczuk, L. Pfeiffer, K. West, ibid. 65, 641 (1990); K. Kheng, R.T. Kox, Y.M. Daubine, F. Bassani, K. Saminadayar, S. Tatarenko, ibid. 71, 1752 (1993); G. Yusa, H. Shtrikman, I. Bar-Joseph, ibid. 87, 216402 (2001); H. Buhmann, L. Mansouri, J. Wang, P.H. Beton, N. Mori, L. Eaves, M. Henini, M. Potemski, Phys. Rev. B 51, 7969 (1995); M. Byszewski, B. Chwalisz, D. K. Maude, M. L. Sadowski, M. Potemski, T. Saku, Y. Hirayama, S. Studenkin, D. G. Austing, A. S. Sachrajda, P. Hawrylak, Nat. Phys. (London) 2, 239 (2006).

[2] S. Glasberg, G. Finkelstein, H. Shtrikman, I. BarJoseph, Phys. Rev. B 59, R10425 (1999).

[3] L. Bryja, A. Wojs, J. Misiewicz, M. Potemski, D. Reuter, A. Wieck, Phys. Rev. B 75, 035308 (2007).

[4] G. Finkelstein, H. Shtrikman, I. Bar-Joseph, Phys. Rev. B 53, 12593 (1996); ibid. 56, 10326 (1997); S. Glasberg, H. Shtrikman, I. Bar-Joseph, ibid. 63, 201308(R) (2001); A.B. Dzyubenko, ibid. 69, 115332 (2004).

[5] D. Yakovlev, V.P. Kochereshko, R.A. Suris, H. Schenk, W. Ossau, A. Waag, G. Landwehr, P.C.M. Christianen, J.C. Maan, Phys. Rev. Lett. 79, 3974 (1997)

[6] B.E. Cole, J.M. Chamberlain, M. Henini, T. Cheng, W. Batty, A. Wittlin, J.A. Parenboom, A. Ardavan, A. Polisski, J. Singelton, Phys. Rev. B 55, 2503 (1997).

[7] A. Wójs, J.J. Quinn, Phys. Rev. B 75, 085318 (2007); A. Wójs, ibid. 76, 085344 (2007). 